\title{
Assessment of the Coronary Artery in High-Pitch, Dual-Source CT Aortography Without ECG Synchronization
}

\author{
Hyeong Gi Choi, ${ }^{1}$ Mi Jung Park,,${ }^{1,}$ Ho Cheol Choi, ${ }^{1}$ Hye Young Choi, ${ }^{1}$ Hwa Seon Shin,,${ }^{1}$ Jae Boem Na, Jae \\ Min Cho, ${ }^{1}$ and Dae Seob Choi ${ }^{1}$ \\ ${ }^{1}$ Department of Radiology, Gyeongsang National University School of Medicine, Gyeongsang National University Hospital, Chilam-Dong, Jinju City, Gyeongnam-Do, Republic \\ of Korea \\ "Corresponding author: Mi Jung Park, MD, Department of Radiology, Gyeongsang National University School of Medicine, Gyeongsang National University Hospital, 90, \\ Chilam-Dong, Jinju, Gyeongnam-Do, Republic of Korea. Tel: +82-557508814, Fax: +82-557581568, E-mail: pichola@naver.com
}

Received 2016 December 22; Revised 2017 March 17; Accepted 2017 April 01.

\begin{abstract}
Background: The high pitch, dual source computed tomography (CT) provides motion-free aortic imaging.

objectives: To evaluate the image quality of coronary artery using dual-source CT aortography without electrocardiogram (ECG) synchronization.

Patients and Methods: One hundred fifty patients ( 87 men; mean age; $63.7 \pm 13.0$ years, mean heart rate; $64.7 \pm 6.6$ beats/min) with suspected or known aortic disease underwent non-ECG gated, high-pitch, dual-source CT aortography. No beta blocker or nitroglycerin was administered for the patients. The image quality of each coronary artery segment and its ostium was graded on a three-point scale (excellent, moderate, and non-diagnostic image quality).

Results: Most patients (88.7\%) showed diagnostic image quality in the ostia of the left main and right coronary artery. Among 1894 coronary arterial segments, 870 (45.9\%) segments were rated as excellent image quality, 507 segments $(26.8 \%)$ were rated as moderate image quality, and 517 segments (27.3\%) were rated as non-diagnostic image quality. In the per-vessel analysis, non-diagnostic image qualities were often found in right coronary artery (44.0\%) and left circumflex artery distribution (36.0\%). In contrast, nondiagnostic image qualities were less frequently found in left main (0.67\%) and left anterior descending distributions (9.9\%).

Conclusion: Non-ECG gated, high-pitch, dual-source CT can be useful to evaluate the coronary ostial involvement in patients with ascending aortic dissection. The left main and left anterior descending coronary arteries exhibit relatively good diagnostic image qualities compared with left circumflex and right coronary arteries.
\end{abstract}

Keywords: Aorta, Coronary Artery Disease, Multidetector Computed Tomography, Cardiac Imaging Techniques, Diagnostic Imaging

\section{Background}

Computed tomography (CT) aortography has been widely used to evaluate aortic disease for initial diagnosis and follow-up. Presence of coronary artery disease (CAD) has been reported in initial diagnosis and follow-up of patients with aortic dissection and aortic aneurysm (1-4) and it has also been reported to affect mortality after surgical repair (5). Furthermore, triple-rule-out CT has been widely used to evaluate the cause of acute chest pain such as aortic disease, pulmonary thromboembolism, and coronary artery disease $(6,7)$. Even though the heart is included in triple-rule-out $\mathrm{CT}$, additional electrocardiogram (ECG)gated, coronary CT is needed for the evaluation of CAD to minimize cardiac motion artifact.

High-pitch CT provides fast volume coverage, high temporal resolution at a low radiation dose in young and adult patients (8-12). Especially, ECG-gated, high-pitch, dualsource CT exhibits good diagnostic performance for evalu- ation of coronary artery disease (9, 12-14). However, coronary CT angiography should be performed carefully, because ECG electrodes must be attached in the anterior thoracic wall, and beta-blockers are necessary to reduce heart rates in patients with high heart rates. Motion-free aortic imaging of the ascending aorta is possible with highpitch dual-source CT without ECG synchronization (11). The left and right coronary arteries arise from the coronary cusp of the aortic root. It is particularly important to evaluate the coronary ostial involvement in patients with ascending aortic dissection. We speculated that the coronary artery is less affected by cardiac motion using high-pitch CT compared with conventional CT.

\section{Objectives}

The purpose of this study was to demonstrate whether high-pitch dual-source CT aortography is feasible for eval- 
uating coronary artery disease without ECG synchronization.

\section{Patients and Methods}

\subsection{Study Population}

One hundred fifty consecutive patients (mean age, 63.7 \pm 13.0 years, 87 men) who underwent CT aortography were included in this study. CT aortography was performed to evaluate suspected aortic dissection $(n=122)$, perioperative evaluation for vascular surgery $(n=20)$, and follow-up for aortic aneurysm $(n=8)$. We excluded the patients with suspected acute coronary syndrome such as ST-elevated myocardial infarction, and elevation of cardiac enzymes. We also excluded patients with heart rates higher than 80 beats/min due to motion artifacts, but we included patients with arrhythmia. We excluded patients with previous hypersensitivity reaction to contrast material and renal failure with high serum creatinine. Premedication such as beta blocker or nitroglycerin was not administered for this study. The patients' heart rates were measured 10 - 20 minutes prior to CT examinations, because the ECG electrode was not attached in the chest wall during conventional CT aortography. The mean heart rate (MHR) was defined as mean value of heart rate during exam and the heart rate variability (HRV) was defined as the difference between the minimum and maximum heart rates. This study was approved by the institutional review board and the written informed consent was waived.

\subsection{CT Parameters}

CT scans were performed with second generation, dual-source CT (Definition Flash; Siemens Medical Systems, Forchheim, Germany). The CT aortography were performed with $120 \mathrm{~mL}$ of contrast media (400 mg iodine/mL, Iomeron, Bracco Imaging SpA, Milan, Italy), followed by 30 $\mathrm{mL}$ of saline solution, both at a flow rate of $4 \mathrm{~mL} / \mathrm{sec}$. A bolus tracking was used in the descending thoracic aorta, and CT was automatically started 12 seconds after the aortic attenuation reached 250 Hounsfield units (HU).

The scan parameters of CT aortography were as follows: slice acquisition, $128 \times 0.6 \mathrm{~mm}$ with a $\mathrm{z}$-flying focal spot; gantry rotation time, 280 milliseconds; pitch, 3.0; tube potential, $100 \mathrm{kV}$; and effective tube current, 320 $\mathrm{mAs} /$ rotation. With the gantry rotation time of 0.28 second, a half-scan reconstruction applied a temporal resolution of 75 milliseconds. The automatic tube current modulation was used to reduce the radiation exposure (Care Dose 4D; Siemens Medical Solutions, Forchheim, Germany). CT scans were performed from clavicular head level to symphysis pubis with craniocaudal direction and a single breath hold during deep inspiration.

Image reconstruction was applied as a slice thickness of $0.75 \mathrm{~mm}$ and an increment of $0.5 \mathrm{~mm}$ with a medium kernel (B26f). The CT raw data were transferred to the commercially available software (Aquarius version 4.4.6.85, Terarecon, San Mateo, California) to generate curved and straight multiplanar reformatted images.

\subsection{Data Readout and Subjective Image Quality Including Mo- tion Artifact}

The 16-segment model, proposed by the American heart association (AHA), was used to assess the segment of coronary artery (15). Each vessel was analyzed in two imaging planes that included one curved multiplanar reformation view and one cross-sectional view. We divided the sixteen coronary artery segments into the following four groups (16): (1) proximal segments (ex, 4 segments levels), including AHA segments 1, 5, 6, 11; (2) the proximal and middle segments of the coronary arteries (ex, 7 segment levels), including AHA segments 1, 2, 5, 6, 7, 11, and 13; (3) 10 segments levels, including AHA segments 1, 2, 3, 4, 5, 6, 7, 8, 11 , and 13; and (4) the complete coronary arterial tree (ex, 16 segment levels), including the total of 16 segments.

Furthermore, we evaluated the coronary ostia with less than $2 \mathrm{~cm}$ distance from the aorta in the left main coronary artery (LMCA) and right coronary artery (RCA). We also assessed the motion artifacts in the ascending aorta. The image quality of the coronary artery and ascending aorta were independently evaluated by two radiologists ( 2 and 7 years of experience in cardiovascular imaging) and the three-point grading scale was used according to the severity of motion artifact. If there were any discrepancy between the two readers, the final decision was reached in consensus. Score 1 was defined as excellent image quality with no motion artifact, score 2 was defined as moderate image quality with mild luminal blurring, and score 3 was defined as a non-diagnostic image quality with severe luminal blurring. We did not consider the other factors that affect the subjective image quality such as severity of calcification and image noise.

3.4. Objective Image Quality Including Image Noise and Contrast-to-Noise Ratio

Contrast-to-noise ratios (CNR) in the LMCA and proximal RCA were measured by two readers independently (17). The region of interest was placed in the contrast enhanced arterial lumen, trying to avoid the atherosclerotic plaque. The vascular contrast was defined as the difference of the mean attenuation between the contrast-enhanced lumen and the adjacent perivascular soft tissue. The image noise 
was measured as the standard deviation of the region of interest in the ascending aorta. CNR was calculated as the vessel contrast divided by the image noise (17).

\subsection{Radiation Dose}

The dose-length product of each patient was obtained from the CT dose report. The effective dose from the CT aortography was estimated based on the dose-length product using a conversion coefficient $\left(\mathrm{k}=0.017 \mathrm{mSv} \times \mathrm{mGy}^{-1} \times\right.$ $\left.\mathrm{cm}^{-1}\right)$ for the aortas of adults $(14,18)$.

\subsection{Statistical Analysis}

The data were analyzed with SPSS software (version 21.0, SPSS Inc., Chicago, Illinois, USA). All data were tested for normality using Shapiro-Wilk tests. Continuous factors were expressed as means \pm SD if distributed normally, otherwise those factors were expressed by medians with the interquartile range. Continuous factors are expressed as the means \pm the standard deviations, and categorical factors are expressed as the frequencies or percentages. A P value $<0.05$ was considered statistically significant. The interobserver agreement for the image qualities was evaluated using Cohen's kappa. The chi-square test was used for categorical data and the unpaired Student's t-test was used for numerical variables.

\section{Results}

\subsection{Motion Artifact}

The patients' characteristics and scan parameters are presented in Table 1 . The interobserver agreement was good regarding the image quality scores. The mean image qualities per segment were $1.83 \pm 0.79$ in the first reader and $1.84 \pm 0.80$ in the second reader. Same scores between two readers were obtained in 1599 of 1894 segments. The Cohen's kappa was $0.755(\mathrm{P}<0.001)$, which indicated good interobserver agreement.

The majority of the patients exhibited no motion artifacts in the ascending aorta. Only one patient exhibited a respiratory motion artifact at the aortic valve level, and a double aortic contour was observed in the ascending aorta. Specifically, this patient exhibited poor image quality in LMCA.

The 1894 coronary arterial segments were visualized in our study (12.7 \pm 1.4 segments per patient; Table 2$)$. Among 1894 segments, 870 (45.9\%) were rated as excellent image quality (score 1 ), 507 segments (26.8\%) were rated as moderate image quality (score 2 ), and 517 segments (27.3\%) were rated as non-diagnostic image quality (score 3 ). Figures 1 and 2 were shown the representative images with excellent
Table 1. Patient Demographics and CT Scan Parameters

\begin{tabular}{|cc}
\hline Variable & $\begin{array}{c}\text { Number }(\%) \text { or } \\
\text { Mean } \pm \\
\text { Standard } \\
\text { Deviation }\end{array}$ \\
\hline Patient demographics & $87(58)$ \\
\hline Male & $63.7 \pm 13.0$ \\
\hline Age & $23.8 \pm 3.2$ \\
\hline Body mass index $\left(\mathrm{kg} / \mathrm{m}^{2}\right)$ & $67.1 \pm 9.5$ \\
\hline Mean heart rate $($ beats $/ \mathrm{min})$ & $12.5 \pm 17.5$ \\
\hline Heart rate variability $($ beats $/ \mathrm{min})$ & \\
\hline CT Scan Parameters & $727.7 \pm 53.6$ \\
\hline Scan range $($ length, $\mathrm{mm})$ & $1.7 \pm 0.1$ \\
\hline Duration of data acquisition $(\mathrm{s})$ & $243.7 \pm 36.4$ \\
\hline Dose length product $(\mathrm{mGy} \times \mathrm{cm})$ & $4.1 \pm 0.6$ \\
\hline Effective radiation dose $(\mathrm{mSv})$ & \\
\hline
\end{tabular}

and non-diagnostic quality scores. The mean image quality score per segment was $1.83 \pm 0.81$. In the per-patient analysis, 14 patients (9.3\%) showed all segments with diagnostic image quality (scores of 1 and 2), and 136 patients (90.7\%) showed at least one segment with non-diagnostic image quality. In the per-patient analysis, the number of segments with non-diagnostic image qualities per patient was $3.0(2.0$ - 4.0) (Figure 3). In the per-vessel analysis, non-diagnostic image qualities were often observed in RCA (44.0\%, 262 of 595 segments) and left circumflex artery (LCX) distributions (36.0\%, 187 of 519 segments). In contrast, non-diagnostic image qualities were observed less frequently in the left main ( $0.67 \%, 1$ of 150 segments) and left anterior descending artery (LAD) distributions (9.9\%, 60 of 608 segments).

We also evaluated the diagnostic image qualities of the segments according to the four levels (Table 3 ). The median numbers of diagnostic segment were $4.0(3.0$ - 4.0) at the 4-coronary segment level, $6.0(5.0-6.0)$ at the 7-segment level, $8.0(6.0$ - 9.0) at the 10-segment level, and 10.0 (8.0 $11.5)$ at the 16-segment level. The percentage of diagnostic segments per evaluated segment was the highest (83.6\%) at the 4-segment level, whereas this value was lowest at the 10-segment (73.2\%) and 16-segment (72.7\%) levels. Furthermore, the percentages of patients with diagnostic image qualities were $60.0 \%$ at the 4 -segment level, $22.7 \%$ at the 7-segment level, $13.3 \%$ at the 10-segment level, and 9.3\% at the 16-segment level. Furthermore, we evaluated the image qualities in the ostia of LMCA and RCA. Most patients ( $88.7 \%, n=133$ ) showed diagnostic image quality in the ostia of both LMCA and RCA. 
Table 2. Subjective Image Quality Scores of Coronary Segments Based on 16 Segments Model

\begin{tabular}{|c|c|c|c|c|c|c|c|c|c|c|c|c|c|c|c|c|}
\hline \multirow{2}{*}{$\begin{array}{l}\begin{array}{l}\text { Vascular } \\
\text { Territory }\end{array} \\
\text { Segment } \\
\text { number }\end{array}$} & \multicolumn{4}{|c|}{ Right Coronary Artery } & \multirow{2}{*}{$\begin{array}{c}\text { Left Main } \\
5\end{array}$} & \multicolumn{5}{|c|}{ Left Anterior Descending Artery } & \multicolumn{5}{|c|}{ Left Circumflex Artery } & \multirow{2}{*}{$\begin{array}{c}\begin{array}{c}\text { Ramus } \\
\text { Intermedius }\end{array} \\
16\end{array}$} \\
\hline & 1 & 2 & 3 & 4 & & 6 & 7 & 8 & 9 & 10 & 11 & 12 & 13 & 14 & 15 & \\
\hline $\begin{array}{l}\text { Evaluated } \\
\text { segments }\end{array}$ & 150 & 150 & 150 & 145 & 150 & 150 & 150 & 150 & 123 & 35 & 148 & 110 & 139 & 77 & 45 & 22 \\
\hline Score 1 & $56(37)$ & $32(21)$ & $51(34)$ & $50(34)$ & $129(86)$ & $123(82)$ & $98(65)$ & $67(45)$ & $68(55)$ & $14(40)$ & $76(51)$ & $37(34)$ & $35(25)$ & $18(23)$ & $9(20)$ & $7(32)$ \\
\hline Score 2 & $41(27)$ & $31(21)$ & $39(26)$ & $33(23)$ & $20(13)$ & $20(13)$ & $40(27)$ & $61(40)$ & $43(35)$ & $14(40)$ & $35(24)$ & $43(39)$ & $45(32)$ & $25(33)$ & $9(20)$ & $8(36)$ \\
\hline Score 3 & $53(36)$ & $87(58)$ & $60(40)$ & $62(43)$ & 1(1) & $7(5)$ & $12(8)$ & $22(15)$ & $12(10)$ & $7(20)$ & $37(25)$ & $30(27)$ & $59(43)$ & $34(44)$ & $27(60)$ & $7(32)$ \\
\hline
\end{tabular}

Table 3. The Number and Percentage of Diagnostic Image Quality According to Coronary Artery Segments of Four Levels ${ }^{\mathrm{a}}$

\begin{tabular}{llll}
\hline Variables & $\begin{array}{l}\text { Number of Diagnostic Segments per } \\
\text { Patient [Median (25-75 Percentile Range) }\end{array}$ & $\begin{array}{l}\text { Number of Diagnostic Segment/Number } \\
\text { of Evaluated Segments (Percentage) }\end{array}$ & $\begin{array}{l}\text { Number of Patients with Diagnostic } \\
\text { Image Quality (Percentage) }\end{array}$ \\
\hline 4 segments level & $4.0(3.0-4.0)$ & $500 / 586(83.6)$ & $90 / 150(60)$ \\
7 segments level & $6.0(5.0-6.0)$ & $892 / 1185(75.3)$ & $34 / 150(22.7)$ \\
\hline 10 segments level & $8.0(6.0-9.0)$ & $1193 / 1630(73.2)$ & $20 / 150(13.3)$ \\
16 segments level & $10.0(8.0-11.5)$ & $1377 / 1894(72.7)$ & $14 / 150(9.3)$ \\
\hline
\end{tabular}

${ }^{a}$ Four segment level indicates AHA segment 1, 5, 6, 11, 7 segments level indicates AHA segment 1, 2, 5, 6, 7, 11, 13, 10 segments level indicates 1, 2, 3, 5, 6, 7, 8, 11, 12, 13, and 16 segments level indicates all sixteen segments.

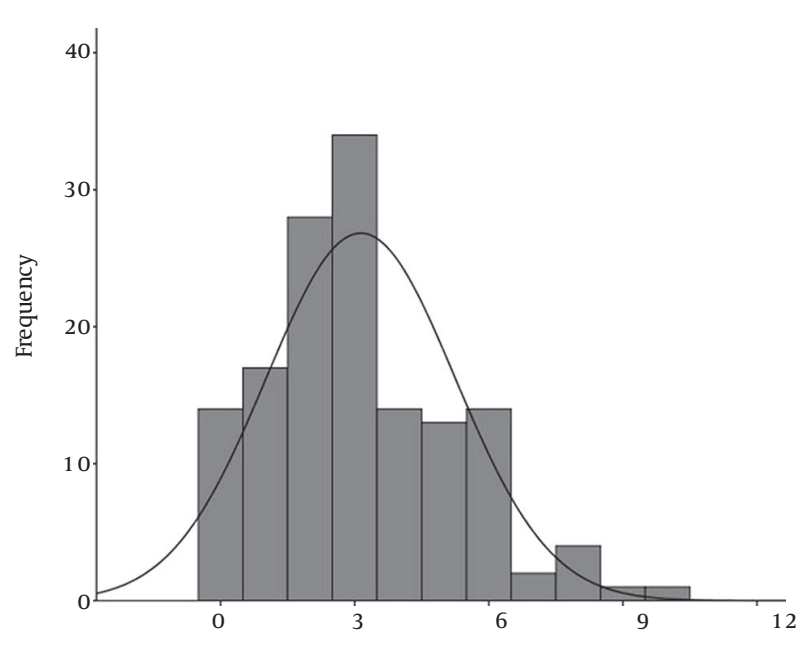

Number of nondiagnostic segments per patient

Figure 1. Number of segments with non-diagnostic image quality per patient

\subsection{Image Noise and CNR}

The interobserver agreements were excellent for the attenuation in LMCA and RCA $(r=0.79 ; \mathrm{P}<0.01)$, the attenuation of the perivascular tissue $(\mathrm{r}=0.73$; $\mathrm{P}<0.01)$, and the standard deviation of the ascending aorta $(\mathrm{r}=0.87 ; \mathrm{P}<$ 0.01). The average image noise was $31.5 \pm 6.7 \mathrm{HU}$ (22.7 - 51.2 $\mathrm{HU})$. The average attenuations of LMCA and RCA were 392.9 $\pm 116.0 \mathrm{HU}$ (204.3 - 631.2 HU) and $388.8 \pm 118.3 \mathrm{HU}$ (268.7 703.6 HU), respectively. The average CNRs of LMCA and RCA

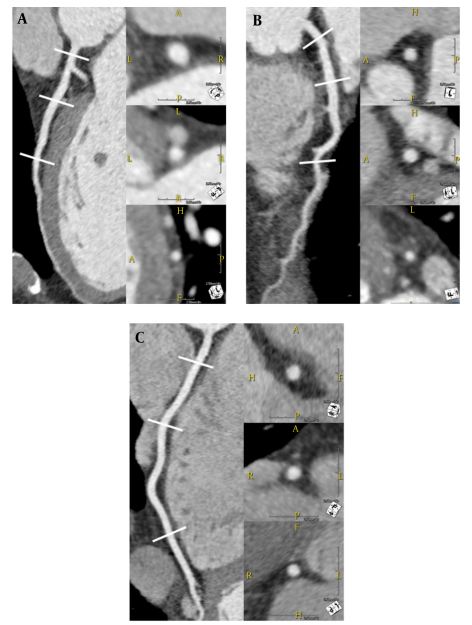

Figure 2. A 50-year- old man with a mean heart rate of 53 beats/min and heart rate variability of 5 beats/min. A-C,Curved multiplanar reformations and cross-sectional images of the left anterior descending (A), left circumflex (B), and right coronary arteries (C) show excellent image quality.

were $29.6 \pm 9.4 \mathrm{HU}(14.2-53.2 \mathrm{HU})$ and $29.0 \pm 8.9(18.5-58.3)$, respectively.

\subsection{Estimation of the Radiation Dose}

The mean dose length product was $243.7 \pm 36.4$ (212 274) $\mathrm{mGycm}$, and the mean effective radiation dose was 4.1 $\pm 0.6(3.6-4.6) \mathrm{mSv}$. 


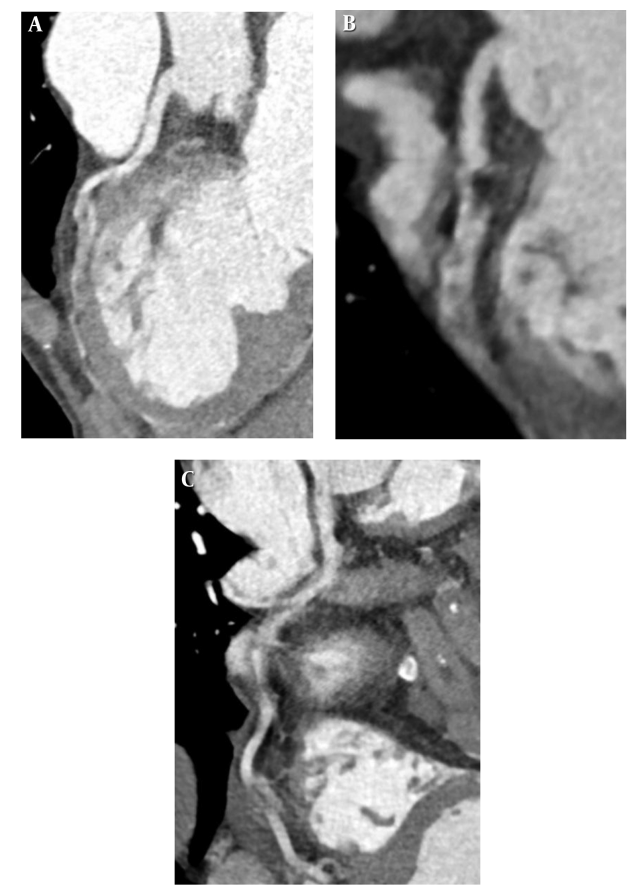

Figure 3. A 67-year- old man with a mean heart rate of 73 beats/min and heart rate variability of 6 beats/min. A-C, Curved multiplanar reformations of the left anterior descending (A), left circumflex (B), and right (C) coronary arteries show nondiagnostic image quality.

\section{Discussion}

Non-ECG gated, low pitch CT is well known to be insufficient for evaluating the ascending aorta and coronary artery due to the cardiac motion artifact (19). Our study demonstrated that the ascending aorta, LMCA and LAD exhibited relatively good diagnostic image qualities compared to LCx and RCA in high pitch, dual source CT aortography. Furthermore, this technique can be useful to evaluate the coronary ostial involvement in patients with ascending aortic dissection.

Prospectively ECG-triggered, high-pitch mode (flash mode) can decrease the estimated radiation dose compared to low pitch mode (19). The high table speed at the pitch of 3.2 is an effective method for achieving entire aorta examination in a single session without ECG synchronization. Furthermore, the high-pitch helical acquisition mode in the dual-source CT enables the gapless image reconstruction. The transitions between sequential images are smooth, so misalignment or stair-step artifacts do not occur. A previous report demonstrated that $100 \mathrm{kVp}$ is sufficient for obtaining the diagnostic image quality of the coronary arteries in patients with low body mass index (BMI) or low weight (10). We excluded the patients with body mass index (BMI) of higher than 30 . We could apply
$100 \mathrm{kVp}$ to reduce the radiation dose. The radiation dose of CT aortography was measured as $4.1 \mathrm{mSv}$ in our study.

Theoretically, cardiac motion artifacts can be reduced by fast scanning, ECG-gating, or postprocessing of the scan. Our study demonstrated that most patients exhibited no motion artifact in the ascending aorta. Only one patient showed a respiratory motion artifact in the ascending aorta level, thus the ascending aorta and LMCA exhibited poor image qualities. It has been reported that non-ECG gated, high-pitch CT can provide motion-free aortic imaging in the ascending aorta $(11,20)$. Furthermore, the evaluation of proximal coronary artery in patients with ascending aortic dissection is important to assess the coronary involvement of dissection flap or the coronary ostium originated from true or false lumen. We found that the majority of patients showed the diagnostic image quality in the ostia of LMCA and RCA. Hence, we expected that highpitch CT angiography(CTA), compared to conventional low pitch CT, could provide the good image quality to evaluate the coronary artery ostial involvement in patients with ascending aortic dissection without additional ECG-gated coronary CT angiography.

High pitch, CT aortography provides good contrast enhancement and objective image quality of the coronary artery. Our study demonstrated a good CNR and low image noise in the coronary artery. Previous studies reported that good contrast enhancement in the coronary artery can be obtained in high-pitch dual-source CTA $(8,20)$. On the other hand, the subjective image quality is not good in high-pitch CT aortography. Our study demonstrated that the LMCA and LAD were less affected by motion artifacts than LCX and RCA. The cause of this phenomenon has been well documented in early studies of coronary artery imaging using electron beam CT. Previous studies demonstrated that motion velocity of the coronary artery is highest in RCA followed by $\operatorname{LCx}$ and $\operatorname{LAD}(21,22)$. Furthermore, the motion of LAD exhibited no significant differences throughout the cardiac cycle, but the motions of LCX and RCA vary significantly throughout the cardiac cycle in patients with heart rates $<80$ beats/min (22). The motion free, cardiac phase was late-systole and mid-diastolic phase in patients with heart rate $<80$ beats/min, but this phase was shortened in the patients with heart rate $>80$ beats/min (22). The similar result was obtained in the previous study using third generation dual source CT (19). These results indicate that non-ECG gated CT does not guarantee the acquisition of motion-free imaging of the whole coronary artery even in high-pitch mode. The majority of patients had at least one segment with non-diagnostic image quality in our study. We found that approximately three segments per patient were of non-diagnostic image quality. In contrast, non-ECG gated, high-pitch CT is sufficient 
for obtaining the diagnostic coronary artery imaging in LMCA and LAD. The CAD in LMCA and proximal LAD are clinically important because significant stenosis in the LMCA and proximal LAD, compared to the other coronary segments, are associated with poor prognosis for cardiovascular event $(23,24)$.

We also analyzed the subjective image qualities in persegment and per-patient analyses according to four levels. The subjective image qualities in our study were poor compared with those of a previous study, using ECG-gated highpitch dual-source coronary CT in patients with heart rate $>70$ beats/min (25), even though we enrolled the patients with heart rate $<80$ beats $/ \mathrm{min}$. Another study analyzed the diagnostic image qualities using non-ECG gated high-pitch dual-source CT in patients with high heart rates (mean heart rate $(\mathrm{MHR})=86$ beats/min). Compared with our results, the diagnostic image qualities in the per-segment analysis were similar at the 4 -segment level ( $88 \%$ vs. $84 \%$ ) and 7-segment level (75\% vs. 75\%) but decreased at the 10segment level (61\% vs. 73\%) and 16-segment level (48\% vs. 73\%) (16). Furthermore, the diagnostic image qualities in the per-segment analysis were similar at the 4-segment level (67\% vs. $60 \%$ ) and 7-segment level (23\% vs. $23 \%$ ) but decreased at the 10-segment level (3\% vs. 13\%) and 16-segment level (0\% vs. 9\%) (16). These results suggest that the image qualities were not hampered at the proximal and midcoronary segment levels but were worsened at the distal coronary segments and branch vessel in patients with high heart rate. Therefore, additional invasive coronary angiography or ECG-gated, coronary CT angiography is needed to evaluate the distal run-off vessel of obstructive CAD, especially for the patients requiring coronary artery bypass graft.

The study limitations are as follows. First, the highpitch CT aortography was performed not for CAD evaluation but for aortic disease evaluation. The diagnostic performance of coronary CT was not verified with invasive coronary angiography. Additionally, beta-blockers or nitroglycerin were not administered in this study. Additional studies are needed to evaluate the diagnostic performance of high-pitch CT aortography. Second, our study only focused on the motion artifact rather than calcification regarding subjective image quality. Calcification is a wellknown factor that reduces the quality of images of the coronary artery (26). Third, the heart rates were measured before CT scan, so the heart rates could have been different during the CT scans. Fourth, we selected the patients with low heart rate and low to moderate BMI. Therefore, the image quality was hampered in case of patients with high heart rate or high BMI.

In conclusion, non-ECG gated, high-pitch dual-source CT can be useful to evaluate the coronary artery ostial in- volvement in patients with aortic dissection. LMCA and LAD were imaged with relatively good diagnostic quality compared with LCx and RCA.

\section{Acknowledgments}

None.

\section{Footnotes}

Authors' Contributions: None declared.

Financial Disclosure: None.

Conflict of Interests: No conflict exists for any of the authors.

Funding/Support: None declared.

\section{References}

1. Creswell LL, Kouchoukos NT, Cox JL, Rosenbloom M. Coronary artery disease in patients with type A aortic dissection. Ann Thorac Surg. 1995;59(3):585-90. doi: 10.1016/0003-4975(94)00880-9. [PubMed: 7887694].

2. Hosokawa Y, Takano H, Aoki A, Inami T, Ogano M, Kobayashi N, et al. Management of coronary artery disease in patients undergoing elective abdominal aortic aneurysm open repair. Clin Cardiol. 2008;31(12):580-5. doi: 10.1002/clc.20335. [PubMed: 19072880].

3. Takigawa M, Yokoyama N, Yoshimuta T, Takeshita S. Prevalence and prognosis of asymptomatic coronary artery disease in patients with abdominal aortic aneurysm and minor or no perioperative risks. Circ J. 2009;73(7):1203-9. doi: 10.1253/circj.CJ-08-1135. [PubMed: 19443955].

4. Yoshikai M, Ikeda K, Itoh M, Noguchi R. Detection of coronary artery disease in acute aortic dissection: the efficacy of 64-row multidetector computed tomography. J Card Surg. 2008;23(3):277-9. doi: 10.1111/j.1540-8191.2008.00594.x. [PubMed: 18435650].

5. Miller JS, Lemaire SA, Coselli JS. Evaluating aortic dissection: when is coronary angiography indicated? Heart. 2000;83(6):615-6. doi: 10.1136/heart.83.6.615. [PubMed: 10814612].

6. Hou DJ, Tso DK, Davison C, Inacio J, Louis LJ, Nicolaou S, et al. Clinical utility of ultra high pitch dual source thoracic CT imaging of acute pulmonary embolism in the emergency department: are we one step closer towards a non-gated triple rule out? Eur J Radiol. 2013;82(10):1793-8. doi: 10.1016/j.ejrad.2013.05.003. [PubMed: 23743054]

7. Halpern EJ. Triple-rule-out CT angiography for evaluation of acute chest pain and possible acute coronary syndrome. Radiology. 2009;252(2):332-45. doi: 10.1148/radiol.2522082335. [PubMed: 19703877].

8. Lell M, Marwan M, Schepis T, Pflederer T, Anders K, Flohr T, et al. Prospectively ECG-triggered high-pitch spiral acquisition for coronary CT angiography using dual source CT: technique and initial experience. Eur Radiol. 2009;19(11):2576-83. doi: 10.1007/s00330-0091558-4. [PubMed:19760421].

9. Goetti R, Feuchtner G, Stolzmann P, Desbiolles L, Fischer MA, Karlo C, et al. High-pitch dual-source CT coronary angiography: systolic data acquisition at high heart rates. Eur Radiol. 2010;20(11):2565-71. doi: 10.1007/s00330-010-1838-z. [PubMed: 20585785]. 
10. Achenbach S, Marwan M, Ropers D, Schepis T, Pflederer T, Anders $\mathrm{K}$, et al. Coronary computed tomography angiography with a consistent dose below $1 \mathrm{mSv}$ using prospectively electrocardiogramtriggered high-pitch spiral acquisition. Eur Heart J. 2010;31(3):340-6. doi: 10.1093/eurheartj/ehp470. [PubMed: 19897497].

11. Beeres M, Schell B, Mastragelopoulos A, Herrmann E, Kerl JM, GruberRouh T, et al. High-pitch dual-source CT angiography of the whole aorta without ECG synchronisation: initial experience. Eur Radiol. 2012;22(1):129-37. doi: 10.1007/s00330-011-2257-5. [PubMed: 21915607].

12. Bridoux A, Hutt A, Faivre JB, Flohr T, Duhamel A, Pagniez J, et al. Coronary artery visibility in free-breathing young children on non-gated chest CT: impact of temporal resolution. Pediatr Radiol. 2015;45(12):1761-70. doi: 10.1007/s00247-015-3401-1. [PubMed: 26293229].

13. Scharf M, Bink R, May MS, Hentschke C, Achenbach S, Uder M, et al High-pitch thoracic CT with simultaneous assessment of coronary arteries: effect of heart rate and heart rate variability on image quality and diagnostic accuracy. JACC Cardiovasc Imaging. 2011;4(6):602-9. doi:10.1016/j.jcmg.2011.02.014. [PubMed: 21679894].

14. Goetti R, Baumuller S, Feuchtner G, Stolzmann P, Karlo C, Alkadhi H, et al. High-pitch dual-source CT angiography of the thoracic and abdominal aorta: is simultaneous coronary artery assessment possible? AJR Am J Roentgenol. 2010;194(4):938-44. doi:10.2214/AJR.09.3482. [PubMed: 20308495].

15. Austen WG, Edwards JE, Frye RL, Gensini GG, Gott VL, Griffith LS, et al A reporting system on patients evaluated for coronary artery disease. Report of the Ad Hoc Committee for Grading of Coronary Artery Disease, Council on Cardiovascular Surgery, American Heart Association. Circulation. 1975;51(4 Suppl):5-40. doi: 10.1161/01.CIR.51.4.5. [PubMed: 1116248].

16. de Malherbe M, Duhamel A, Tacelli N, Hachulla AL, Pontana F, Faivre JB, et al. Ultrafast imaging of the entire chest without ECG synchronisation or beta-blockade: to what extent can we analyse the coronary arteries? Insights Imaging. 2012;3(1):73-9. doi: 10.1007/s13244-011-01330. [PubMed: 22696000].

17. Lee SK, Jung JI, Ko JM, Lee HG. Image quality and radiation exposure of coronary CT angiography in patients after coronary artery bypass graft surgery: influence of imaging direction with 64-slice dual-source CT. J Cardiovasc Comput Tomogr. 2014;8(2):124-30. doi: 10.1016/j.jcct.2013.12.011. [PubMed: 24661825].

18. Macari M, Chandarana H, Schmidt B, Lee J, Lamparello P, Babb J. Ab- dominal aortic aneurysm: can the arterial phase at CT evaluation after endovascular repair be eliminated to reduce radiation dose? $\mathrm{Ra}$ diology. 2006;241(3):908-14. doi: 10.1148/radiol.2413051571. [PubMed: 17065562].

19. Sandfort V, Ahlman MA, Jones EC, Selwaness M, Y. Chen M, R. Folio $\mathrm{L}$, et al. High pitch third generation dual-source CT: Coronary and cardiac visualization on routine chest CT. J Cardiovasc Comput Tomogr. 2016;10(4):282-8. doi:10.1016/j.jcct.2016.03.007. [PubMed: 27133589].

20. Lell M, Hinkmann F, Anders K, Deak P, Kalender WA, Uder M, et al. High-pitch electrocardiogram-triggered computed tomography of the chest: initial results. Invest Radiol. 2009;44(11):728-33. doi: 10.1097/RLI.0b013e3181b9df7e. [PubMed: 19809339].

21. Achenbach S, Ropers D, Holle J, Muschiol G, Daniel WG, Moshage W. In-plane coronary arterial motion velocity: measurement with electron-beam CT. Radiology. 2000;216(2):457-63. doi: 10.1148/radiology.216.2.ro0au19457. [PubMed: 10924570].

22. Lu B, Mao SS, Zhuang N, Bakhsheshi H, Yamamoto H, Takasu J, et al. Coronary artery motion during the cardiac cycle and optimal ECG triggering for coronary artery imaging. Invest Radiol. 2001;36(5):2506. doi: 10.1097/00004424-200105000-00002. [PubMed: 11323512].

23. Klein LW, Weintraub WS, Agarwal JB, Schneider RM, Seelaus PA, Katz RI, et al. Prognostic significance of severe narrowing of the proximal portion of the left anterior descending coronary artery. Am J Cardiol. 1986;58(1):42-6. doi:10.1016/0002-9149(86)90238-9. [PubMed: 3728330].

24. Andreini D, Pontone G, Mushtaq S, Bartorelli AL, Bertella E, Antonioli L, et al. A long-term prognostic value of coronary CT angiography in suspected coronary artery disease. JACC Cardiovasc Imaging. 2012;5(7):690-701. doi:10.1016/j.jcmg.2012.03.009. [PubMed: 22789937].

25. Pansini V, Remy-Jardin M, Tacelli N, Faivre JB, Flohr T, Deken V, et al. Screening for coronary artery disease in respiratory patients: comparison of single- and dual-source CT in patients with a heart rate above 70 bpm. Eur Radiol. 2008;18(10):2108-19. doi: 10.1007/s00330008-0998-6. [PubMed:18463871].

26. Park MJ, Jung JI, Choi YS, Ann SH, Youn HJ, Jeon GN, et al. Coronary CT angiography in patients with high calcium score: evaluation of plaque characteristics and diagnostic accuracy. Int J Cardiovasc Imaging. 2011;27 Suppl 1:43-51. doi: 10.1007/s10554-011-9970-7. [PubMed: 22048849]. 